\title{
Tuberculosis among Children and Adolescents at HIV Treatment Centers in Sub-Saharan Africa
}

Anna M. Mandalakas, ${ }^{1}$ Alexander W. Kay, ${ }^{1}$ Jason M. Bacha, Tara Devezin,

Rachel Golin, Katherine R. Simon, Dilsher Dhillon, Sandile Dlamini, Andrew DiNardo,

Mogo Matshaba, Jill Sanders, Lineo Thahane, Pauline M. Amuge, Saeed Ahmed, Moorine P. Sekadde,

Neway G. Fida, Bhekumusa Lukhele, Nodumo Chidah, David Damba, Joseph Mhango,

Moses Chodota, Makhorong Matsoso, Angelina Kayabu, Richard S. Wanless, Gordon E. Schutze

\section{$\underset{\text { MDUCATION }}{\text { Medsce ACTIVITY }}$}

In support of improving patient care, this activity has been planned and implemented by Medscape, LLC and Emerging Infectious Diseases. Medscape, LLC is jointly accredited by the Accreditation Council for Continuing Medical Education (ACCME), the Accreditation Council for Pharmacy Education (ACPE), and the American Nurses Credentialing Center (ANCC), to provide continuing education for the healthcare team.

Medscape, LLC designates this Journal-based CME activity for a maximum of 1.00 AMA PRA Category 1 Credit(s) ${ }^{\mathrm{TM}}$. Physicians should claim only the credit commensurate with the extent of their participation in the activity.

Successful completion of this CME activity, which includes participation in the evaluation component, enables the participant to earn up to $1.0 \mathrm{MOC}$ points in the American Board of Internal Medicine's (ABIM) Maintenance of Certification (MOC) program. Participants will earn MOC points equivalent to the amount of CME credits claimed for the activity. It is the CME activity provider's responsibility to submit participant completion information to ACCME for the purpose of granting ABIM MOC credit.

All other clinicians completing this activity will be issued a certificate of participation. To participate in this journal CME activity: (1) review the learning objectives and author disclosures; (2) study the education content; (3) take the post-test with a 75\% minimum passing score and complete the evaluation at http://www.medscape.org/journal/eid; and (4) view/print certificate. For CME questions, see page 3121.

Release date: November 20, 2020; Expiration date: November 20, 2021

Learning Objectives

Upon completion of this activity, participants will be able to:

- Describe TB incidence and period prevalence among children and adolescents with HIV (C/ALHIV), according to an analysis of data from 7 integrated pediatric HIV/TB centers in 6 countries in sub-Saharan Africa

- Determine TB outcomes and risk factors among C/ALHIV, according to an analysis of data from 7 integrated pediatric HIV/TB centers in 6 countries in sub-Saharan Africa

- Identify clinical implications of the association between antiretroviral therapy and TB prevalence and risk factors for adverse TB outcomes among C/ALHIV, according to an analysis of data from 7 integrated pediatric HIV/TB centers in 6 countries in sub-Saharan Africa

\section{CME Editor}

Deanna Altomara, BA, Copyeditor, Emerging Infectious Diseases. Disclosure: Deborah Wenger, MBA, has disclosed no relevant financial relationships.

CME Author

Laurie Barclay, MD, freelance writer and reviewer, Medscape, LLC. Disclosure: Laurie Barclay, MD, has disclosed no relevant financial relationships.

\section{Authors}

Disclosure: Anna M. Mandalakas, MD, PhD; Alexander W. Kay, MD; Jason M. Bacha, MD, MS; Tara Devezin, BA, MID; Rachel Golin, MD, MHS; Katherine R. Simon, MD; Dilsher Dhillon, MS; Sandile Dlamini, BA; Andrew DiNardo, MD; Mogomotsi Matshaba, MB, BCh, BAO; Jill E. Sanders, MD; Lineo Thahane, MD; Pauline M. Amuge, MBChB, MMed; Saeed Ahmed, MD; Moorine P. Sekadde, MMed, MPH; Neway G. Fida, MD; Bhekumusa Lukhele, PhD; Nodumo Chidah, MBA; David Damba, MS; Joseph Mhango, MS; Moses Chodota, BA; Makhorong Matsoso, BSc; Angelina Kayabu, BSc; and Gordon E. Schutze, MD, have disclosed no relevant financial relationships. Richard Sebastian Wanless, MBChB, PhD, has disclosed the following relevant financial relationships: served as an advisor or consultant for Bristol-Myers Squibb Foundation; INNOCIMAB PTE. LTD.

${ }^{1}$ These authors contributed equally to this article. 
HIV-infected children and adolescents are at increased risk for tuberculosis (TB). Antiretroviral therapy (ART) reduces TB risk in HIV-infected adults, but its effectiveness in HIV-infected children and adolescents is unknown. We analyzed data from 7 integrated pediatric HIV/TB centers in 6 countries in sub-Saharan Africa. We used a Bayesian mixed-effect model to assess association between ART and TB prevalence and used adaptive lasso regression to analyze risk factors for adverse TB outcomes. The study period encompassed 57,525 patient-years and 1,160 TB cases (2,017 cases/100,000 patient-years). Every 10\% increase in ART uptake resulted in a $2.33 \%$ reduction in TB prevalence. Favorable TB outcomes were associated with increased time in care and early ART initiation, whereas severe immunosuppression was associated with death. These findings support integrated HIV/TB services for HIV-infected children and adults and demonstrate the association of ART uptake with decreased TB incidence in high HIV/TB settings.

$\mathrm{T}$ uberculosis (TB) is an underestimated cause of death in children (1); it is accurately diagnosed and reported in only $45 \%$ of children with the disease (2). When accounting for underdetection, the World Health Organization (WHO) estimated that, in 2017, a total of 1.12 million TB cases developed in infants, children, and adolescents $\leq 14$ years of age and 1.60 million cases in adolescents and young adults 15-24 years of age (2). WHO also estimated that TB was associated with 205,000 deaths in children, including 32,000 in HIV-infected children and adolescents; these deaths account for $13 \%$ of total TB-associated deaths in HIV-infected persons, although only $5 \%$ of HIV-infected persons are children (2). Children might be at increased risk for TB because they receive antiretroviral therapy (ART) at lower rates than adults. According to the Joint United Nations Programme on HIV/AIDS (3), only $53 \%$ of eligible children worldwide received ART in 2019.

Author affiliations: Texas Children's Hospital, Houston, Texas, USA (A.M. Manadalakas, A.W. Kay, J.M. Bacha, T. Devezin, K.R. Simon, D. Dhillon, S. Dlamini, A. DiNardo, M. Matshaba, J. Sanders, L. Thahane, S. Ahmed, N. Chidah, D. Damba, M. Chodota, M. Matsoso, A. Kayabu, R.S. Wanless, G.E. Schutze); Baylor College of Medicine, Houston (A.M. Manadalakas, A.W. Kay, J.M. Bacha, T. Devezin, D. Dhillon, A. DiNardo, B. Lukhele, R.S. Wanless, G.E. Schutze); Baylor College of Medicine Children's Foundation Swaziland, Mbabane, Swaziland (A.W. Kay, S. Dlamini, B. Lukhele); Baylor College of Medicine Children's Foundation Tanzania, Mbeya, Tanzania (J.M. Bacha); US Agency for International Development, Washington, DC, USA (R. Golin); Baylor College of Medicine Children's Foundation Malawi, Lilongwe, Malawi (K.R. Simon, S. Ahmed, J. Mhango);
Before ART was widely available, TB incidence and TB-related deaths were substantially higher among HIV-infected children and adolescents than among peers without HIV (4). Multiple studies have demonstrated declines in TB incidence among this group after ART scale-up initiatives $(5,6)$. A metaanalysis of data from children estimated a pooled hazard ratio of 0.3 (95\% CI $0.21-0.39$ ) and declining TB risk for 2 years after ART initiation $(5,7)$. However, TB remains a major cause of illness and death in children receiving ART (8).

Although risk factors for TB and adverse TB outcomes are well-documented among HIV-infected adults (9), risk factors among HIV-infected children and adolescents are poorly understood, particularly since the 2016 recommendations for universal ART for all HIV-infected persons (10). Some systematic reviews suggest that immunosuppression predicts TB incidence (7) among HIVinfected children and adolescents. Studies examining data sourced from a single country typically demonstrate that 1 or 2 risk factors, such as age $<2$ years, extrapulmonary TB, malnutrition, severe immunosuppression, WHO HIV clinical stage, or TB treatment status (11-14) can predict death among children with HIV-associated TB. Large or multinational studies of TB risk factors and outcomes among HIV-infected children and adolescents are few $(15,16)$ and urgently needed.

Limited and conflicting data exist on outcomes among HIV-infected children and adolescents in whom TB developed before versus after ART initiation. In a multinational cohort of children from predominantly resource-limited countries, no association existed between TB outcomes and disease onset relative to ART initiation (15). In contrast, other studies have demonstrated lower death rates among children on ART at the time of TB diagnosis (17). WHO

Technical Support to PEPFAR Programs in the Southern Africa Region, Lilongwe (K.R. Simon, S. Ahmed, J. Mhango); Botswana-Baylor Children's Clinical Centre of Excellence, Gaborone, Botswana (M. Matshaba, N. Chidah); Baylor College of Medicine Children's Foundation Lesotho, Maseru, Lesotho (J. Sanders, L. Thahane, M. Matsoso); Baylor College of Medicine Children's Foundation Uganda, Kampala, Uganda (P.M. Amuge, D. Damba); National Tuberculosis and Leprosy Programme, Kampala, Uganda (M.P. Sekkade); US Agency for International Development, Pretoria, South Africa (N.G. Fida); Baylor College of Medicine Children's Foundation Tanzania, Mwanza, Tanzania (M. Chodota, A. Kayabu)

DOI: https://doi.org/10.3201/eid2612.202245 
recommends that HIV-infected children and adolescents start ART as soon as possible and within $\leq 8$ weeks of beginning TB treatment (18). Recent evidence derived from the same multinational cohort demonstrates that this recommendation was poorly implemented; only $46 \%$ of ART-naive children began treatment within $\leq 8$ weeks of starting TB treatment. However, when implemented successfully, this measure was associated with favorable TB treatment outcomes (64\% vs. $40 \%$; $p=0.04)(15)$.

Data regarding TB incidence, management, and outcomes among HIV-infected children and adolescents are mostly sourced from single healthcare centers, limiting their generalizability. We analyzed these variables in the largest reported multinational study of TB in HIV-infected children and adolescents in countries in sub-Saharan Africa.

\section{Materials and Methods}

\section{Strengthening the Reporting of Observational Studies in Epidemiology Statement}

This study was conducted in accordance with the Strengthening the Reporting of Observational Studies in Epidemiology guidelines (19). Our primary objectives were to estimate longitudinal TB incidence within the context of increasing ART coverage and identify risk factors for death from TB in HIV-infected children and adolescents in various stages of ART.

\section{Participants}

We examined TB outcomes of HIV-infected children and adolescents receiving care at 7 treatment centers (Centers of Excellence; COEs) within the Baylor International Pediatric AIDS Initiative at Texas Children's Hospital network, spanning 6 countries: Botswana, Swaziland, Lesotho, Malawi, Tanzania (locations in Mbeya and Mwanza), and Uganda. To avoid sampling bias, we included data from all children receiving care during from January 2013 through June 2017.

\section{Outcomes of Interest}

We used TB case and outcome definitions from WHO (20) (Appendix Table 1, https://wwwnc.cdc.gov/ EID/article/26/12/20-2245-App1.pdf). We categorized TB outcomes as favorable (cured or treatment completed), unfavorable (death), lost to follow-up (LTFU), or transferred out of the COE.

\section{Data Extraction}

We analayzed data from Janaury 2013 through June 2017 from the electronic medical records of HIV-infected children and adolescents $\leq 19$ years of age. The deidentified data from the 7 COEs enables follow-up of individual patients for longitudinal analysis.

The duration of each COE's study period depended on when that $\mathrm{COE}$ began collecting TB data using electronic medical records. Data collection began in 2013 at all COEs (except for Malawi, which began data collection in 2016) and continued through June 2017.

\section{Statistical Approach}

We calculated the annual TB incidence for all HIVinfected children and adolescents at each COE. We modeled the TB incidence as a function of time, ART uptake, and COE. We included a random intercept for the COE, enabling us to visualize how ART uptake varied by COE and by year. We ran this model under a Bayesian framework using $\mathrm{R}$ with the library brms (21). We used a similar Bayesian mixed-effect model to determine association between isoniazid preventive therapy (IPT) use and TB incidence (22). Data on IPT use were available from 4 COEs: Swaziland, Tanzania-Mbeya, Tanzania-Mwanza, and Uganda. We considered children to be eligible for IPT if they were $>12$ months of age and had not previously received IPT.

We examined variables in bivariate analysis if $>75 \%$ of data were available. We excluded some variables, such as specific anthropometrics (e.g., height, mid-upper arm circumference) and Mycobacterium bovis BCG vaccination status because of missing data. We considered CD4 values and viral loads if measured $\leq 60$ days before or after the analytic baseline. For bivariate analysis, we used a $\chi^{2}$ test for categorical independent variables and a Wilcoxon rank-sum test for continuous independent variables. We used multinomial logistic regression to study the univariate association between age and outcome. To relax the assumption of age having a constant effect on outcome, we modeled age using natural cubic splines, using the splines package in R, with 3 knots at equally spaced quantiles. To determine age-related risk for death we calculated the instantaneous rate of change using the method of finite differences.

We used an adaptive lasso logistic regression model to examine the association between TB outcome and 13 independent variables (23). Adaptive lasso normalizes coefficients; therefore, no reference level is preselected. Instead, the model minimizes the influence of coefficients unassociated with the outcome; these coefficients set the reference levels for the remaining coefficients. We selected the penalty parameter to minimize deviance using leave-oneout cross-validation; we used the selected penalty to 
fit the final model. We included risk factors that we hypothesized, a priori, would affect TB outcomes (Appendix Table 2). We conducted the adaptive lasso procedure using library glmnet in R (23) and postselection inference of the selected coefficients using library selective Inference, also in R (24). We developed 3 models to examine associations with favorable outcomes (cure or treatment completion) against death. We examined the outcome within all participants, participants on ART at time of TB diagnosis, and participants not on ART at diagnosis. We conducted sensitivity analyses of HIV-infected children and adolescents who were LTFU or died. We developed additional models evaluating outcomes categorized by $\mathrm{WHO}$ as favorable (cure or treatment completion) or unfavorable (death, LTFU, or not evaluated/transferred out) (Appendix Tables 3-5).

\section{Ethics Statement}

All clinical investigation supporting the data handling, analysis, and reporting of these findings was conducted according to the principles expressed in the Declaration of Helsinki. Approval was obtained from all necessary ethics bodies in each country (i.e., the Baylor College of Medicine Children's Foundation or Trust, the national ethics committee in each country, and the Baylor College of Medicine Institutional Review Board).

\section{Results}

\section{TB Incidence}

We analyzed data on 1,160 HIV-infected children and adolescents in whom TB was diagnosed during the study period, which encompassed 57,525 patientyears. During the 4 -year study period, overall TB incidence was 2,017 cases/100,000 patient-years (range 454 cases/100,000 patient-years in Botswana to 4,385 cases/100,000 patient-years in Tanzania-Mwanza). These incidences were substantially higher than those estimated by WHO for the general populations of the respective countries (Table 1 ).
The age distribution of the cohort was similar across sites. TB incidence was highest among infants and children $<5$ years of age but was elevated among school-aged children, a trend that persisted into early and late adolescence (Figure 1). Increasing age was associated with more favorable outcomes. Children $\leq 7$ years of age had a higher risk for death than schoolaged children (i.e. 8-10 years of age) and adolescents (i.e., 11-19 years of age) (Figure 2, panels A, B). Of patients receiving TB treatment, $32 \%$ had TB infection confirmed by bacteriologic testing, usually GeneXpert (Cepheid, https://www.cepheid.com); this percentage excludes children at the Lesotho COE because it had incomplete data. Confirmation rates ranged from $24 \%$ for infants and children $\leq 2$ years of age to $51 \%$ for adolescents 10-19 years of age.

Throughout the study, rates of ART administration increased and the prevalence of TB declined at most sites (Figure 3, panels A, B). For every 10\% increase in the number of HIV-infected children and adolescents who received ART, the overall prevalence of TB in the clinical network decreased $2.33 \%$ (95\% credible interval 0.58\%-4.4\%) (Figure 3, panel C).

We also observed an increase in the number of eligible children starting IPT. Average rates of IPT use across all COEs increased from $8.68 \%$ in 2014 to $27.5 \%$ in 2017 . When we limited our analysis to the 4 COEs with available IPT data, we observed no effect on TB prevalence $(0.4 \%$ increase, $95 \%$ credible interval $-0.3 \%$ to $1.0 \%$ ) associated with each $10 \%$ increase in the number of HIV-infected children and adolescents receiving IPT.

\section{TB Outcomes and Risk Factor Analysis}

Most children and adolescents had favorable outcomes: across all sites, $75 \%$ (95\% CI 67\%-87\%) of patients, including those who were LTFU or had transferred out, had favorable outcomes (Table 2). On average, children with favorable TB outcomes had received care at the clinics nearly a year longer than children who died $(\mathrm{p}<0.01)$. Ten percent $(95 \%$

\begin{tabular}{|c|c|c|c|c|}
\hline \multirow[b]{2}{*}{ Country } & \multirow{2}{*}{$\begin{array}{c}\text { TB incidence } \\
\text { no. per } 100,000 \text { patient- } \\
\text { years }(95 \% \mathrm{Cl})\end{array}$} & \multicolumn{2}{|c|}{$\begin{array}{c}\text { WHO } 2017 \text { country estimates, } \\
\text { no. per } 100,000 \text { persons }(95 \% \mathrm{Cl})\end{array}$} & \multirow{2}{*}{$\begin{array}{c}\text { TB incidence/WHO } 2017 \\
\text { TB country estimate } \\
\text { fold difference }\end{array}$} \\
\hline & & TB & HIV-associated TB & \\
\hline Botswana & $454(299-608)$ & $300(232-376)$ & $144(93-206)$ & 1.5 \\
\hline Eswatini & $2,612(2,205-3,020)$ & 308 (236-389) & $213(138-304)$ & 8.5 \\
\hline Lesotho & $3,762(3,376-4,148)$ & 665 (430-949) & $470(298-680)$ & 5.6 \\
\hline Malawi & 1,159 (791-1,528) & $131(70-210)$ & $65(42-93)$ & 8.8 \\
\hline Tanzania-Mwanza & $4,385(3,747-5,024)$ & 269 (127-464) & $84(54-120)$ & 16.3 \\
\hline Tanzania-Mbeya & $3,995(3,498-4,492)$ & $269(127-464)$ & $84(54-120)$ & 14.8 \\
\hline Uganda & $656(546-766)$ & $201(118-305)$ & $80(52-114)$ & 3.2 \\
\hline
\end{tabular}

WHO, World Health Organization. 

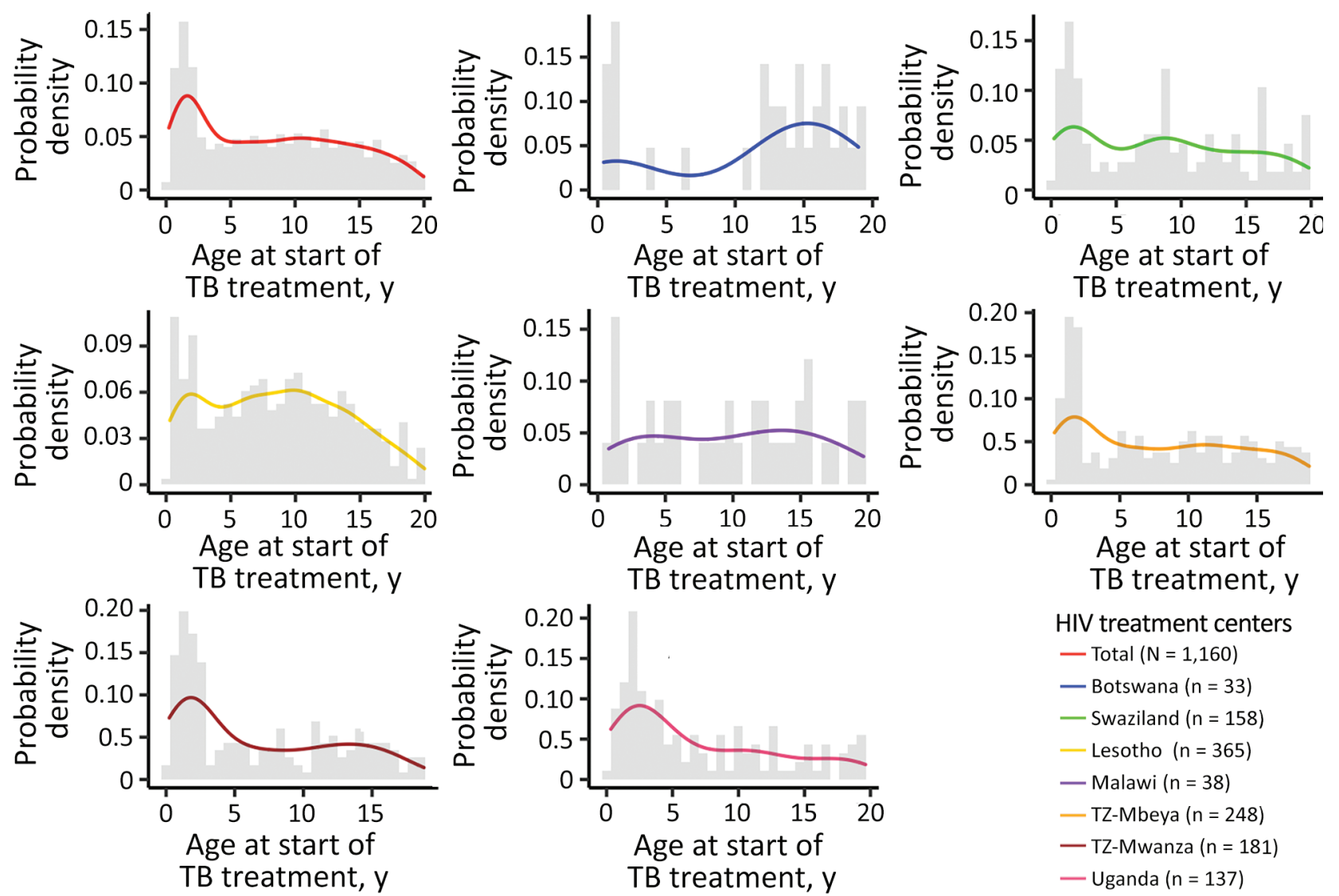

Figure 1. Incidence of tuberculosis (TB) among HIV-infected children and adolescents, 2013-2017. The age at start of TB treatment is plotted as a smoothed line and histogram against the probability of TB diagnosis on the basis of the prevalence of that age within the overall cohort of HIV-infected children and adolescents. The data are presented combined and stratified by HIV treatment center.

CI 5\%-15\%) of HIV-infected children and adolescents with TB died. If we assumed all HIV-infected children and adolescents who were LTFU died, the death ratio would increase to $13 \%$ (95\% CI 6\%-20\%).

We used bivariate analysis to identify factors associated with TB outcome (Table 2). Extrapulmonary disease increased the odds of death $(\mathrm{p}<0.01)$. The death ratio for patients who had previously been treated for TB was similar to the death ratio for patients who had not, even if we assumed all patients
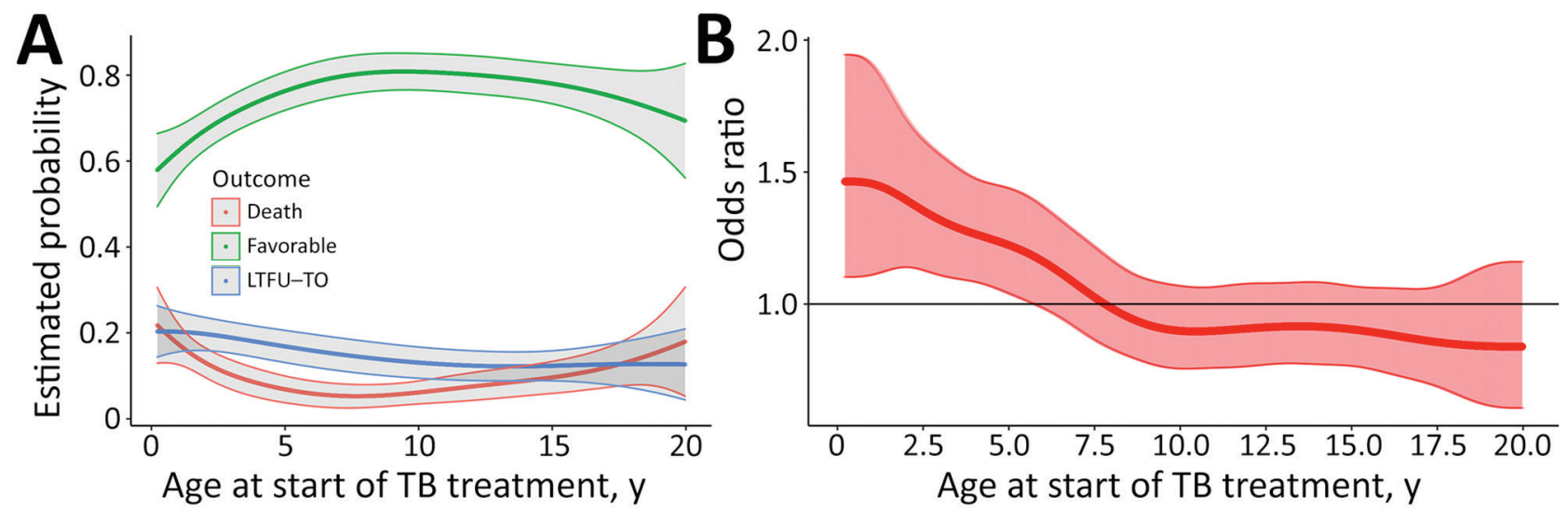

Figure 2. Probabilities of specific outcomes for TB in HIV-infected children and adolescents, 2013-2017. A) Probability (with 95\% Cls) of outcomes stratified by age at start of TB treatment. B) Instantaneous odds ratios for death at each age. The odds ratio reflects the change in odds of death according to age at start of TB treatment. LTFU-TO, lost to follow-up or transferred out; TB, tuberculosis. 
who were LTFU died. HIV-infected children and adolescents who had engaged in facility-based HIV care for more time were more likely to have favorable outcomes $(\mathrm{p}<0.01)$.

HIV-infected children and adolescents who never started ART were less likely than those in all other groups to have a favorable outcome (Figure 4, panel A). HIV-infected children and adolescents who began ART during the 6 months before the start of their TB treatment had the highest death ratio (14\%); children who had never been on ART but began it within 8 weeks of TB diagnosis had the lowest death ratio $(8 \%)$. Immune status was highly predictive of death

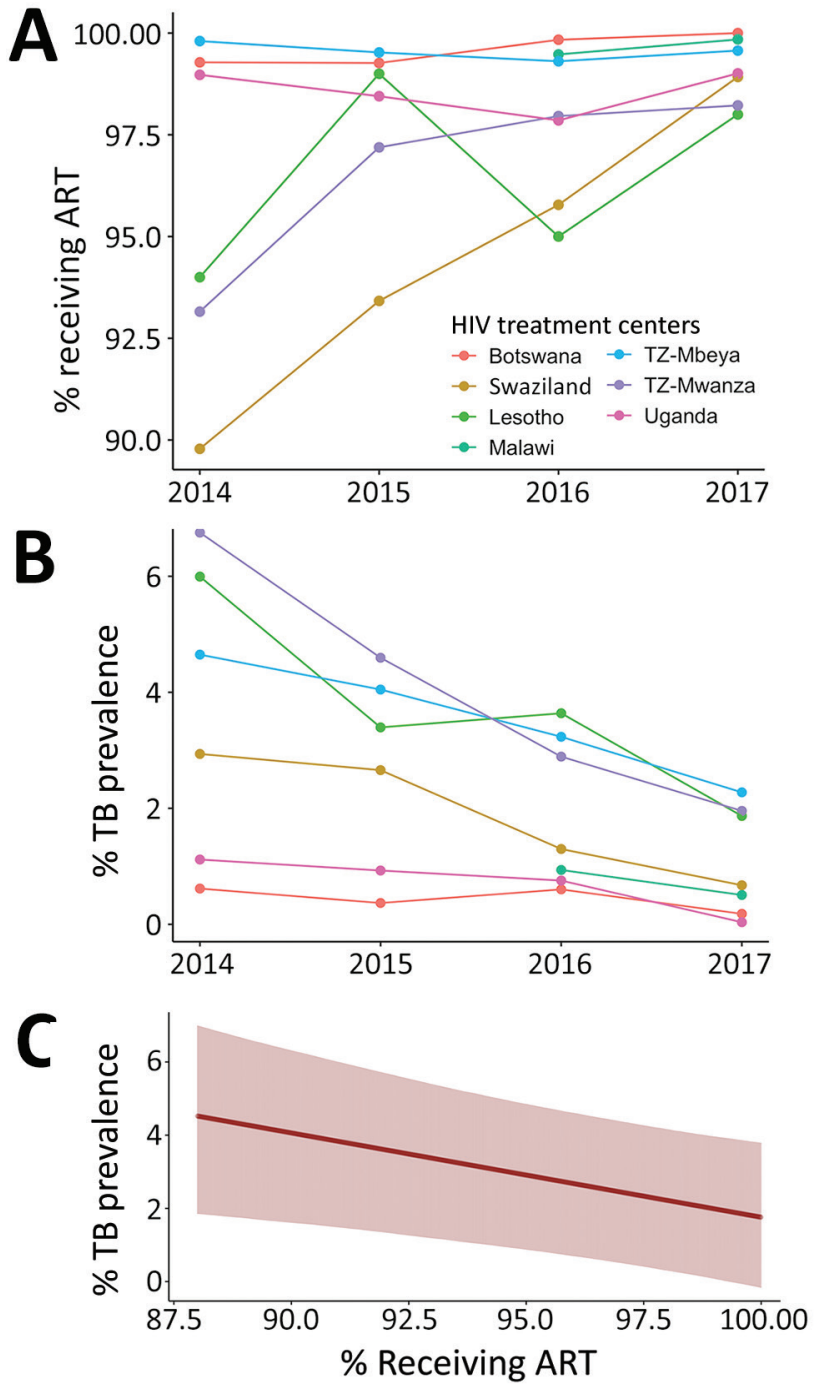

Figure 3. ART use and TB prevalence in HIV-infected children and adolescents, 2013-2017. A) Annual percentage of the cohort at each HIV treatment center receiving ART. B) Annual percentage of the cohort at each treatment center in whom TB was diagnosed. C) Declining TB prevalence with increase in ART uptake, averaged across all treatment centers in the study period. ART, antiretroviral therapy; TB, tuberculosis.
(Figure 4, panel B). HIV-infected children and adolescents with severe immunosuppression had $>5$-fold increased odds of death compared with those without immune suppression ( $16 \%$ vs. $3 \%$; $p<0.01$ ); we defined severe immunosuppression as a CD4 percentage of $<25 \%$ in children $<5$ years of age or CD4 count $<200$ cells $/ \mathrm{mm}^{3}$ in children $\geq 5$ years of age.

We developed 3 multivariate models to comprehensively examine associations with favorable outcomes against death, considering a patient's history of ART. The model comparing all 1,029 patients considered 11 factors (Table 3 ). This model demonstrated the influence of immune status at the time of TB diagnosis, showing that HIV-infected children and adolescents without immune suppression (CD4 percentage $>30 \%$ in children $<5$ years of age or CD4 count $>350$ cells $/ \mathrm{mm}^{3}$ in children $\geq 5$ years of age) at TB diagnosis had a $58 \%$ lower odds of death (odds ratio [OR] 0.42 [95\% CI 0.13-0.94]; $\mathrm{p}=0.04$ ) than children with advanced immune suppression. In addition, patients who had never been on ART but received it $\leq 8$ weeks after TB diagnosis had a $59 \%$ lower odds of death than those who received ART $>8$ weeks after TB diagnosis (OR 0.41 [95\% CI 0.14-0.60]; p<0.01). The multivariate analysis did not demonstrate an increased odds of death for HIV-infected children and adolescents who started ART in the 6 months before TB diagnosis.

The model comparing the 597 patients who had received ART before TB diagnosis considered 13 factors (Table 4). Children with severe immunosuppression at TB diagnosis had a 4 times higher odds of death than children with advanced immune suppression (OR 4.29 [95\% CI 1.23-29.28]; $p=0.03$ ). A patient's odds of death increased with each advance in WHO stage at TB diagnosis (OR 2.18 [95\% CI 1.915.98]; $\mathrm{p}<0.01)$.

The final model of patients who had never received ART, prior to TB diagnosis, comprised 391 patients with favorable outcomes and 41 patients who died; this model was intractable and did not converge. Results evaluating associations with programmatic outcomes were similar to the results described in the previous paragraphs (Appendix Tables 3-5).

\section{Discussion}

TB is the leading cause of death in HIV-infected persons (2). This multicountry study of TB in HIVinfected children and adolescents revealed high TB incidences that greatly exceeded estimated population level TB incidences of all individual countries represented by the cohort. Consistent with recent systematic reviews and meta-analyses (7), our evidence demonstrates that although ART significantly reduc- 
Table 2. Bivariate analysis of associations with TB treatment outcomes in HIV-infected children and adolescents, 2013-2017*

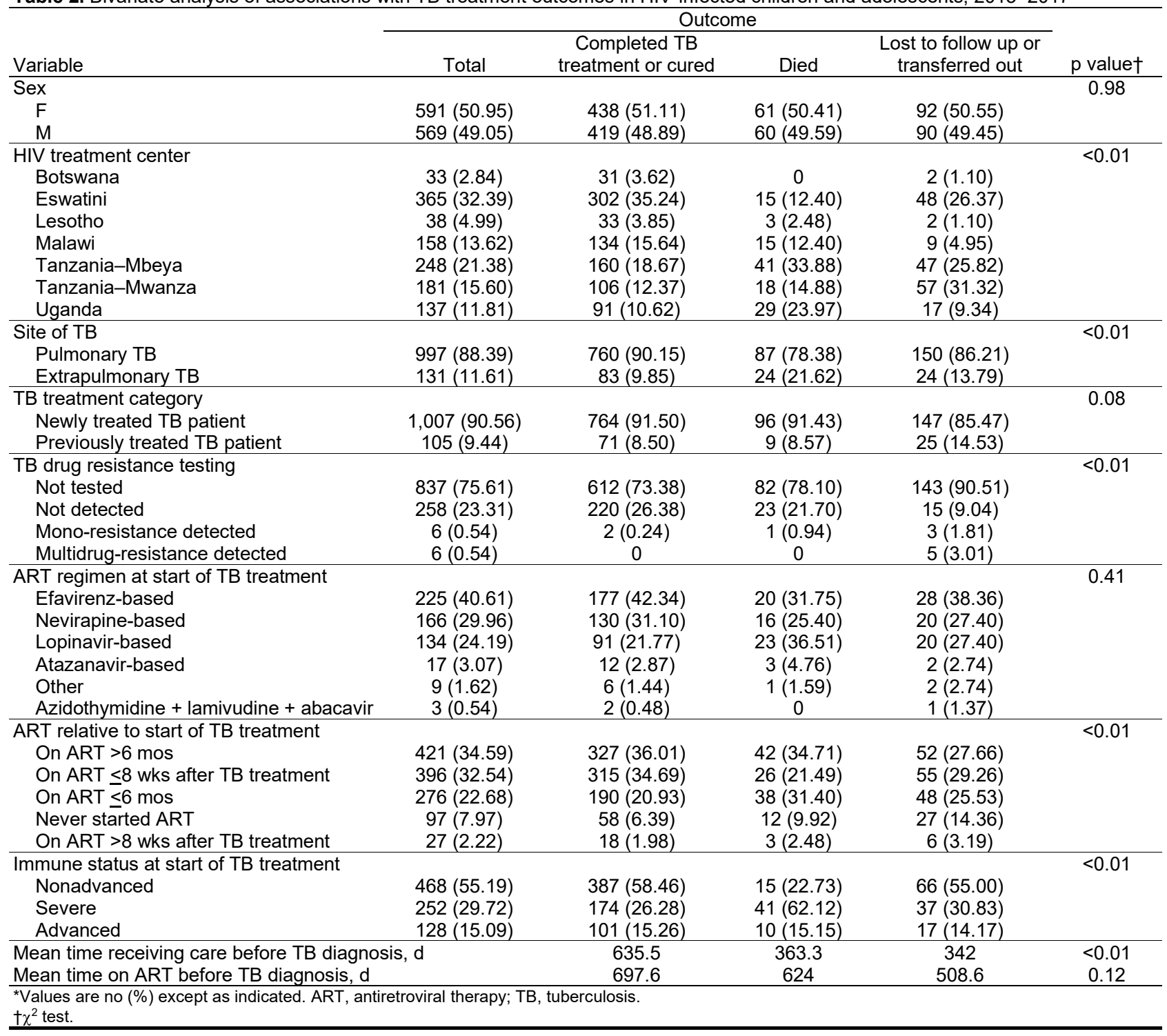

es the prevalence of TB in HIV-infected children and adolescents, the risk for TB remains elevated even among a population with excellent ART coverage. TBrelated deaths decreased in adults during 1996-2011, when ART use increased in various countries (25). Although we cannot ascribe causality between increasing ART coverage and declining TB prevalence, this association is notable given the very high initial ART coverage in our study. Increasing ART uptake $>90 \%$ was associated with ongoing declines in TB incidence, suggesting that the Joint United Nations Programme on HIV/ AIDS 95-95-95 targets for HIV might also reduce TB incidence. Likewise, HIV-infected children and adolescents with favorable TB outcomes had received care for nearly a year longer, on average, than children who died. These findings highlight the importance of early HIV diagnosis, prompt treatment, and patient retention in HIV-infected children and adolescents.

Of HIV-infected children and adolescents with TB, 32\% had a confirmed TB diagnosis; the rate of TB confirmation increased with age. This high rate of disease confirmation is consistent with prior studies, which have found that HIV infection does not significantly reduce the likelihood of disease confirmation in children (26-28). However, other multinational cohorts have reported lower rates of disease confirmation in HIV-infected children and adolescents, a discrepancy that might reflect the greater testing capacity at the COEs (15). Most HIV clinics treating children in sub-Saharan Africa do not have the capacity to collect TB specimens in children (29). Confirmatory 


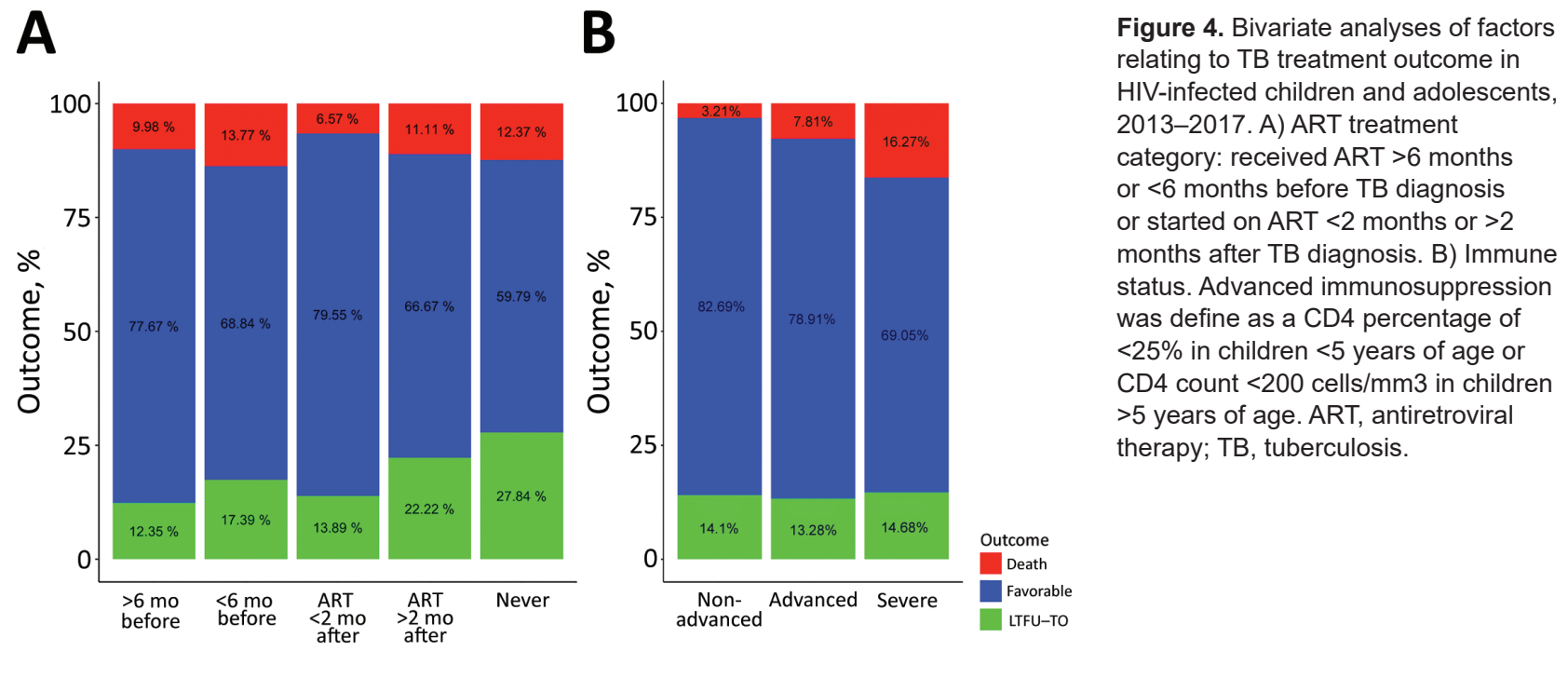

diagnostic testing remains a challenge more broadly across low and middle-income countries in sub-Saharan Africa; furthermore, current testing strategies are invasive and extremely uncomfortable, necessitating the development of child-friendly diagnostic tools.

Within the cohort, HIV-infected children and adolescents $<5$ years of age had the greatest risk for $\mathrm{TB}$, a finding consistent with existing literature (14). Likewise, children in this age range were more likely to die (4). However, TB incidence did not decline significantly among school-age children $\leq 10$ years of age, an age group in HIV-negative cohorts that has a significantly reduced risk for TB (30). This analysis, like others (31), suggests that HIV reduces the protective effect of age on TB risk.

Overall, 75\% of HIV-infected children and adolescents in this cohort had favorable treatment outcomes, whereas $10 \%$ died. Existing literature estimates that in sub-Saharan Africa, $16 \%$ of HIV-infected children and adolescents who are LTFU die, regardless of TB status (32). Because $4 \%$ of patients in our study were LTFU, the death rate of our cohort might be closer to $10 \%-13 \%$. Recent evidence estimates a cumulative all-cause death rate of $3 \%$ at 3 months, $5 \%$ at 6 months, $6 \%$ at 12 months, and $7 \%$ at 24 months after ART initiation in HIV-infected children and adolescents in sub-Saharan Africa (33).

Approximately $60 \%$ of $\mathrm{HIV}$-infected children and adolescents have severe immunosuppression at TB diagnosis (13). In contrast, $33 \%$ of children in this cohort had advanced or severe immunosuppression at TB diagnosis. Severe immunosuppression was associated with up to a 4-fold higher risk for death than advanced immunosuppression. Similarly, advanced WHO stage (noted before TB diagnosis) was associated with a 2-fold increased risk for death. This finding highlights the need for scale-up of HIV identification and treatment methods, such as family index

\begin{tabular}{|c|c|c|}
\hline Variable & Odds ratio $(95 \% \mathrm{Cl})$ & $p$ value \\
\hline \multicolumn{3}{|l|}{ Country } \\
\hline Swaziland, Lesotho, Malawi, Tanzania-Mbeya, Tanzania-Mwanza, and Uganda & Referent & \\
\hline Botswana & $0.09(0.00-22.83)$ & 0.1811 \\
\hline \multicolumn{3}{|l|}{ TB drug resistance } \\
\hline Not tested, multidrug resistance, and not detected & Referent & \\
\hline Monoresistance & $18.11(0.00-31,381.00)$ & 0.4642 \\
\hline \multicolumn{3}{|l|}{ ART category } \\
\hline $\begin{array}{l}\text { On ART }>6 \text { mos before TB treatment, on ART }<6 \text { mos before TB treatment, } \\
\text { and on } A R T>8 \text { ws after starting TB treatment }\end{array}$ & Referent & \\
\hline Never on ART & $3.38(0.21-20.02)$ & 0.2168 \\
\hline On ART $\leq 8$ wks after TB treatment & $0.41(0.14-0.60)$ & $0.0051 \dagger$ \\
\hline \multicolumn{3}{|l|}{ Immune status } \\
\hline Advanced & Referent & \\
\hline Not advanced & $0.42(0.13-0.94)$ & $0.0412 \dagger$ \\
\hline Severe & $1.88(0.70-4.80)$ & 0.1233 \\
\hline Each increasing WHO stage & $3.64(2.50-7.17)$ & $<0.001 \ddagger$ \\
\hline
\end{tabular}


TB Among Children at HIV Treatment Centers

Table 4. Predictors of favorable TB outcomes against death in HIV-infected children and adolescents on ART at TB diagnosis, 2013-2017

\begin{tabular}{lc}
\hline Variable & Odds ratio (95\% Cl) \\
\hline Country & $\mathrm{p} \mathrm{value}$ \\
$\quad$ Swaziland, Lesotho, Malawi, Tanzania-Mbeya, Tanzania-Mwanza, and Uganda & Referent \\
$\quad$ Botswana & $0.22(0.01-1.90)$ \\
\hline TB drug resistance & Referent \\
$\quad$ Not tested, multidrug resistance, not detected & 0.0858 \\
$\quad$ Mono INH resistance & $23.64(0.00-28,630.46)$ \\
\hline Immune status & 0.3917 \\
$\quad$ Advanced & Referent \\
$\quad$ Not advanced & $0.59(0.12-5.17)$ \\
$\quad$ Severe & $4.29(1.23-29.28)$ \\
\hline Each increasing WHO stage & $2.18(1.91-5.98)$ \\
\hline${ }^{*}$ Significant result $(\mathrm{p}<0.05)$ & 0.2767 \\
\hline
\end{tabular}

testing, provider-initiated testing and counseling, Test and Start models (10), and prompt ART initiation. Public health officials must further evaluate the care of children and adolescents with advanced HIV to develop strategies that promote survival (34).

The bivariate and multivariate analyses demonstrate a dramatic reduction of death in children who started ART within $\leq 8$ weeks after TB diagnosis. Multivariate analysis shows that starting ART within $\leq 8$ weeks after beginning TB treatment was associated with a $59 \%$ reduction in death compared with children on ART before their TB diagnosis or beginning it $>8$ weeks after starting TB treatment. Furthermore, children who never initiated ART had a $>3$ times higher risk for death than those who were on ART before their TB diagnosis or began ART $>8$ weeks after starting TB treatment. We need more data to ascertain whether ART can further reduce HIV-associated TB death in children when initiated within $\leq 2$ weeks after TB treatment. Most HIV-infected children and adolescents with TB have paucibacillary disease, which can inhibit confirmatory TB testing and might reduce children's risk for immune reconstitution syndrome after starting ART. Furthermore, children have lower risk for adverse reactions to $\mathrm{TB}$ treatment and ART than adults. Thus, early simultaneous initiation of ART and TB treatment might be a safer treatment strategy in children. Starting ART at the same time as TB treatment might reduce the number of HIV-infected children and adolescents with TB who are LTFU before starting ART. This promising treatment strategy should be evaluated in controlled studies.

Among HIV-infected children and adolescents who were not on ART at TB diagnosis, $85 \%$ started ART within $\leq 8$ weeks of beginning TB treatment, confirming that this intervention is attainable in a setting with high HIV/TB prevalence. Children who started ART $\leq 8$ weeks after TB treatment had the lowest odds of death, even lower than those of children on ART at TB diagnosis. We hypothesize that many children who develop TB while on ART are not virologically surpressed and are therefore more likely to die from this disease. The bivariate analysis, but not the multivariate analysis, indicated an increased odds of death among children who began ART $\leq 6$ months before TB diagnosis. This finding suggests that HIV-infected children and adolescents with unmasking TB-immune reconstitution syndrome, an exaggerated inflammatory manifestation of TB during early ART, might have an increased odds of death. Prospective studies evaluating time from TB diagnosis to ART initiation has benefitted the treatment of adults with HIV (35). Similar prospective trials are needed to inform treatment of HIV-infected children and adolescents.

This study has some limitations. Because we used data from clinical settings, missing data precluded analysis of some variables. Nevertheless, this evidence is representative of well-managed clinics in countries in sub-Saharan Africa with consistent access to diagnostic technology and ART medications. We identified an association between ART and TB prevalence but cumulative IPT coverage also increased over time across a subset of COEs. We did not observe an association between IPT uptake and TB prevalence. This lack of association might have been caused by sample size limitations, inconsistent IPT availability, or heterogeneity of IPT guidelines; therefore, we cannot draw strong conclusions about the effects of IPT on TB prevalence. Because only a subset of COEs provided data on IPT, we did not include it in the TB outcomes models. We could not compare annual incidence of TB at the clinic level with national trends because of recent changes in WHO's estimation methods (36). Last, as with all retrospective analysis, the possibility exists for inaccurate entry of clinical data. We attempted to limit this source of error through manual data checks. 
The strong association between immune suppression at TB diagnosis and death highlights the importance of early TB case detection and ART initiation among HIV-infected children and adolescents. Furthermore, we found a strong association between favorable TB outcome and increased length of time in care. These associations emphasize the importance of early HIV case detection and prompt ART initiation. Finally, we found an association between increased ART coverage and decreased TB incidence, as well as ART initiation within $\leq 8$ weeks after starting TB treatment and favorable outcomes. Collectively, these findings support the continued need to promote policies and implement practices that fully integrate optimal HIV and TB treatment in countries with high burdens of these diseases.

\section{Acknowledgments}

We thank the local Ministry of Health officials, the Baylor teams, children, adolescents, and families across the Baylor International Pediatric AIDS Initiative at Texas Children's Hospital network who contributed to this analysis.

This work is in memory of Peter Nicholas Kazembe (1955-2020), who made monumental contributions to the health of children and families in Malawi and beyond.

This study was supported by the US President's Emergency Plan for AIDS Relief through the US Agency for International Development (USAID) award no. AID-674-A-16-00003. In addition to USAID funding, Texas Children's Hospital provided funds to support this research and disseminate findings.

The contents in this manuscript are those of the authors and do not necessarily reflect the view of the US Agency for International Development, the U.S. President's Emergency Plan for AIDS Relief, or the United States Government. This manuscript was made possible by the support of the American people through the United States Agency for International Development under the President's Emergency Plan for AIDS Relief. The contents in this article are the sole responsibility of the authors, and do not necessarily reflect the views of USAID, PEPFAR or the United States Government. USAID Award Number AID-674-A-16-00003.

\section{About the Author}

Dr. Mandalakas is the director of the Global Tuberculosis Program at Texas Children's Hospital. Her research interests include child TB and TB/HIV.

\section{References}

1. Dodd PJ, Yuen CM, Sismanidis C, Seddon JA, Jenkins HE. The global burden of tuberculosis mortality in children: a mathematical modelling study. Lancet Glob Health. 2017;5:e898-906. https://doi.org/10.1016/ S2214-109X(17)30289-9

2. World Health Organization. Global tuberculosis report. 2018 [cited 2020 Mar 20]. https:/ / www.who.int/tb/publications / global_report/gtbr2018_main_text_28Feb2019.pdf

3. UNAIDS. Global HIV \& AIDS statistics - 2020 fact sheet. 2020 [cited 2020 Aug 1]. https:/ / www.unaids.org/en/ resources / fact-sheet\#: :text $=38.0 \% 20$ million $\% 20 \% 5 B 31.6 \% 20$ million \%E2\%80\%9344.5,AIDS\%2Drelated \%20illnesses $\% 20$ in $\% 202019$

4. Jenkins HE, Yuen CM, Rodriguez CA, Nathavitharana RR, McLaughlin MM, Donald P, et al. Mortality in children diagnosed with tuberculosis: a systematic review and meta-analysis. Lancet Infect Dis. 2017;17:285-95. https://doi.org/10.1016/S1473-3099(16)30474-1

5. Bakeera-Kitaka S, Conesa-Botella A, Dhabangi A, Maganda A, Kekitiinwa A, Colebunders R, et al. Tuberculosis in human immunodeficiency virus infected Ugandan children starting on antiretroviral therapy. Int J Tuberc Lung Dis. 2011;15:1082-6. https://doi.org/10.5588/ijtld.10.0538

6. Martinson NA, Moultrie H, van Niekerk R, Barry G, Coovadia A, Cotton M, et al. HAART and risk of tuberculosis in HIV-infected South African children: a multi-site retrospective cohort. Int J Tuberc Lung Dis. 2009;13:862-7.

7. Dodd PJ, Prendergast AJ, Beecroft C, Kampmann B, Seddon JA. The impact of HIV and antiretroviral therapy on TB risk in children: a systematic review and meta-analysis. Thorax. 2017;72:559-75. https:/ / doi. org/10.1136/thoraxjnl-2016-209421

8. B-Lajoie MR, Drouin O, Bartlett G, Nguyen Q, Low A, Gavriilidis G, et al. Incidence and prevalence of opportunistic and other infections and the impact of antiretroviral therapy among HIV-infected children in low- and middle-income countries: a systematic review and meta-analysis. Clin Infect Dis. 2016;62:1586-94. https:// doi.org/10.1093/cid/ciw139

9. Nguyen DT, Jenkins HE, Graviss EA. Prognostic score to predict mortality during TB treatment in TB/HIV co-infected patients. PLoS One. 2018;13:e0196022. https:/ / doi.org/10.1371/journal.pone.0196022

10. World Health Organization. Consolidated guidelines on the use of antiretroviral drugs for treating and preventing HIV infection: recommendations for a public health approach. 2016 [cited 2020 Sep 22]. https:/ / apps.who.int/iris/ bitstream/handle/10665/208825/9789241549684_eng.pdf;js essionid =C22AB630B33C95684E57DA59D7B9F56D? sequence $=1$

11. Atalell KA, Birhan Tebeje N, Ekubagewargies DT. Survival and predictors of mortality among children co-infected with tuberculosis and human immunodeficiency virus at University of Gondar Comprehensive Specialized Hospital, Northwest Ethiopia. A retrospective follow-up study. PLoS One. 2018;13:e0197145. https:/ / doi.org/10.1371/ journal.pone.0197145

12. Hicks RM, Padayatchi N, Shah NS, Wolf A, Werner L, Sunkari VB, et al. Malnutrition associated with unfavorable outcome and death among South African MDR-TB and HIV co-infected children. Int J Tuberc Lung Dis. 2014;18:1074-83. https://doi.org/10.5588/ijtld.14.0231

13. Buck WC, Olson D, Kabue MM, Ahmed S, Nchama LK, Munthali A, et al. Risk factors for mortality in Malawian children with human immunodeficiency virus and tuberculosis co-infection. Int J Tuberc Lung Dis. 2013;17:1389-95. https:// doi.org/10.5588/ijtld.13.0030

14. Wiseman CA, Schaaf HS, Cotton MF, Gie RP, Jennings T, Whitelaw A, et al. Bacteriologically confirmed tuberculosis 
in HIV-infected infants: disease spectrum and survival. Int J Tuberc Lung Dis. 2011;15:770-5. https:/ / doi.org/10.5588/ ijtld.10.0501

15. Carlucci JG, Blevins Peratikos M, Kipp AM, Lindegren ML, Du QT, Renner L, et al.; International Epidemiology Databases to Evaluate AIDS (IeDEA) Network. Tuberculosis treatment outcomes among HIV/TB-coinfected children in the International Epidemiology Databases to Evaluate AIDS (IeDEA) Network. J Acquir Immune Defic Syndr. 2017; 75:156-63. https://doi.org/10.1097/QAI.0000000000001335

16. Osman M, Lee K, Du Preez K, Dunbar R, Hesseling AC, Seddon JA. Excellent treatment outcomes in children treated for tuberculosis under routine operational conditions in Cape Town, South Africa. Clin Infect Dis. 2017;65:1444-52. https://doi.org/10.1093/cid/cix602

17. Walters E, Cotton MF, Rabie H, Schaaf HS, Walters LO, Marais BJ. Clinical presentation and outcome of tuberculosis in human immunodeficiency virus infected children on anti-retroviral therapy. BMC Pediatr. 2008;8:1. https://doi.org/10.1186/1471-2431-8-1

18. World Health Organization. Consolidated guidelines on the use of antiretroviral drugs for treating and preventing HIV infection: recommendations for a public health approach second edition. 2016 [cited 2020 Mar 24]. https:/ / www.who. int/hiv/pub/arv/arv-2016

19. von Elm E, Altman DG, Egger M, Pocock SJ, Gøtzsche PC, Vandenbroucke JP; STROBE Initiative. The Strengthening the Reporting of Observational Studies in Epidemiology (STROBE) statement: guidelines for reporting observational studies. Lancet. 2007;370:1453-7. https://doi.org/10.1016/ S0140-6736(07)61602-X

20. World Health Organization. Definitions and reporting framework for tuberculosis - 2013 revision. 2013 [cited 2020 Mar 24]. https:/ / www.who.int/tb/publications/ definitions/en

21. Bürkner PC. brms: an R package for Bayesian multilevel models using Stan. J Stat Softw. 2017;80:1-28. https:/ / doi.org/ 10.18637/jss.v080.i01

22. Goodrich B, Gabry J, Ali I, Brilleman S. rstanarm: Bayesian applied regression modeling via Stan. R package. Version 2174 [cited 2020 Mar 20]. http:/ / mc-stanorg/2018

23. Friedman J, Hastie T, Tibshirani R. Regularization paths for generalized linear models via coordinate descent. J Stat Softw. 2010;33:1-22. https://doi.org/10.18637/jss.v033.i01

24. Tibshirani R, Tibshirani R, Taylor J, Loftus J, Reid S, Markovic J. Selective inference: tools for post-selection inference. 2019 [cited 2020 Mar 20]. https:/ / cran.rproject.org/web/packages/selectiveInference/selective Inference.pdf

25. Yan I, Bendavid E, Korenromp EL. Antiretroviral treatment scale-up and tuberculosis mortality in high TB/ HIV burden countries: an econometric analysis. PLoS One. 2016;11:e0160481. https://doi.org/10.1371/ journal.pone.0160481

26. Connell TG, Zar HJ, Nicol MP. Advances in the diagnosis of pulmonary tuberculosis in HIV-infected and
HIV-uninfected children. J Infect Dis. 2011;204(Suppl 4):S1151-8. https://doi.org/10.1093/infdis/jir413

27. Zar HJ, Workman L, Isaacs W, Dheda K, Zemanay W, Nicol MP. Rapid diagnosis of pulmonary tuberculosis in African children in a primary care setting by use of Xpert MTB/RIF on respiratory specimens: a prospective study. Lancet Glob Health. 2013;1:e97-104. https:/ / doi.org/ 10.1016/S2214-109X(13)70036-6

28. Detjen AK, DiNardo AR, Leyden J, Steingart KR, Menzies D, Schiller I, et al. Xpert MTB/RIF assay for the diagnosis of pulmonary tuberculosis in children: a systematic review and meta-analysis. Lancet Respir Med. 2015;3:451-61. https://doi.org/10.1016/S2213-2600(15)00095-8

29. Reid MJ, Saito S, Fayorsey R, Carter RJ, Abrams EJ. Assessing capacity for diagnosing tuberculosis in children in sub-Saharan African HIV care settings. Int J Tuberc Lung Dis. 2012;16:924-7. https:/ / doi.org/10.5588/ijtld.11.0816

30. Seddon JA, Chiang SS, Esmail H, Coussens AK. The wonder years: what can primary school children teach us about immunity to Mycobacterium tuberculosis? Front Immunol. 2018;9:2946. https:// doi.org/10.3389/fimmu.2018.02946

31. Marais BJ, Graham SM, Cotton MF, Beyers N. Diagnostic and management challenges for childhood tuberculosis in the era of HIV. J Infect Dis. 2007;196(Suppl 1):S76-85. https://doi.org/10.1086/518659

32. Braitstein P, Songok J, Vreeman RC, Wools-Kaloustian KK, Koskei P, Walusuna L, et al. "Wamepotea" (they have become lost): outcomes of HIV-positive and HIV-exposed children lost to follow-up from a large HIV treatment program in western Kenya. J Acquir Immune Defic Syndr. 2011;57: e40-6. https://doi.org/10.1097/QAI.0b013e3182167f0d

33. U.S. President's Emergency Plan for AIDS Relief. PEPFAR 2019 country operational plan guidance for all PEPFAR countries. 2019 [cited 2020 Apr 20]. https:/ / www.state. gov/wp-content/uploads/2019/08/PEPFAR-Fiscal-Year2019-Country-Operational-Plan-Guidance.pdf

34. World Health Organization. Package of care for children and adolescents with advanced HIV disease: stop AIDS. 2020 [cited 2020 Jul 10]. https:/ / www.aidsdatahub.org/sites/ default/files/resource/who-package-care-children-andadolescents-advanced-hiv-disease-2020.pdf

35. Havlir DV, Kendall MA, Ive P, Kumwenda J, Swindells S, Qasba SS, et al.; AIDS Clinical Trials Group Study A5221. Timing of antiretroviral therapy for HIV-1 infection and tuberculosis. N Engl J Med. 2011;365:1482-91. https:/ / doi.org/10.1056/NEJMoa1013607

36. García-Basteiro AL, Brew J, Williams B, Borgdorff M, Cobelens F. What is the true tuberculosis mortality burden? Differences in estimates by the World Health Organization and the Global Burden of Disease study. Int J Epidemiol. 2018;47:1549-60. https://doi.org/10.1093/ije/dyy144

Address for correspondence: Anna M. Mandalakas, Baylor College of Medicine, 1102 Bates St, Ste 630, Houston, TX 77030, USA; email: anna.mandalakas@bcm.edu 